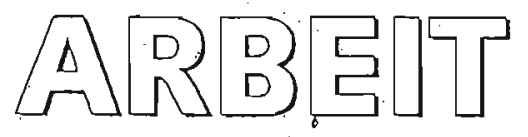

Zeltschrift für Arbeltsforschung Arbeltsgestaltung und Arbeitspolitik

\section{Jahirgang (2002) Heft 4}

Lucius \& Lucius Verlagsgeśs. mbH . Gerokstr. 51 - 70184 Stuttgart (Geschäftsführer: Dr. Wulf D. v. Lúcius) Fax 0711/242088. eMail: lucius@luciusverlag.com · http://www.lúciusverlag.com Herausgeber: Dr. Monika Goldmann, Dortmund; Dr. Hermann Groß, Köln; Prof. Dr. Hermann Kotthoff,
Saarbrucken: Prof. Dr. Hartmut Neuendorf, Dormund; Prof. Dr. Angela Paul-Kohlhoff, Darmstadt; Dr. Gerd

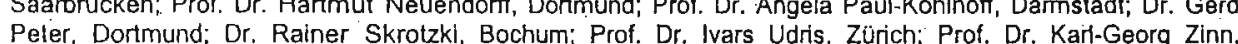
Peler, Dort
Aachen.

Unter Mitwirkung von: Prof. Dr. Hansjürgen Daheim, Köln; Prof. Dr. Christian von Ferber, Düsseldorf; Dr. Rüdiger Klatt, Dortmund; Prof. Dr. Kan Krahn, Blelefeld; Prof. Dr. Rainer Müller, Bremen; Prof. Dr. Ursula - Müller, Bielefeld; Prof. Dr. Hildegard Marla Nickel, Berlin; Prof, Dr. Willi Pöhler, Remagen; Andreas Renner, Düsseldorf; Prof. Dr. Eva Senghaas-Knobioch, Bremen; Prof. Dr. Eberhard Ulichi Zürich; Dr. Volker Volkholz, Dortmund;

Redaktion: Martina Riezler (Koordination), Ingrid Goertz (Sekr.), Dr. Gerd Peter, Olaf Katenkamp (sfs) Renate Schneider, Bettina Müller (ISO)

Redaktions-Anschrlft: Landesinstitut Sozialforschungsstelle, Evinger Platz 17, D-44339 Dortmund, Tel. 0231/8596241; Fax 0231/8596100. Bitte senden Sie redaktionelle Zuschriften und Manuskripte nur an diese Anschrift. sfs: E-mall: goentz@sfs-dortmund.de, riezler@sís-dortmund.de.

Intemet: http://mww.sis-dortmund de; http://mww zeitschrift-arbeit de

Rezensionsredaktions-Anschrift: Institut zur Erforschung sozialer Chancen, Kuenstraße 18, D-50733 Köln. Tel. 0221/97304336; Fax 0221/97304310. Bitte senden Sie Rezensionsexemplare und Korrespondenz, die Rezensionen betrifft, an diese Anschrift. Unverlangt eingesandte Rezensionsexemplare können nich zurückgesandt werden. ISO: E-mail: renate.schneider@iso-koeln.de. Intemet: http://www. iso-koeln.de.

Bezugsbedingungen: Jährlich erscheinen 4 Hefte.

Jahresabonnement 2002: $\in 58,-/ \mathrm{sFr}$ 96,-, für Studierende gegen Studienbescheinigung $\in 42,-1 \mathrm{sFr} 73$,-, jeweils inkl. MwSt. Einzeiheft $€ 18$, - Nersandkosten Inland $€ 4,-/ \mathrm{sFr} 7,50$; Ausland $€ 8,-1 \mathrm{sFr} 15$,-

Alle Bezugspreise und Versandkosten unterliegen der Preisbindung. Abbestellungen müssen spätestens 3 Monate vor Ende des Kalenderjahres schriftlich beim Verlag erfolgen.

Abonnentenverwaltung (zuständig für Neubestellungen, Adressänderungen und Reklamationen) bltte direkt an die Verlagsauslieferung: Brockhaus/Commisslon . Postfach . D - 70803 Komwestheim

Tel. 07154/1327-37, FAX 07154/1327-13. eMail: lucius@brocom:de

Anzelgenverwaltung beim Veriag (Anschrift wie oben). Es gilt die Anzeigenpreisliste vom 1. Januar 2002. () 2002 Lucius \& Lucius Verlagsges. mbH, Stuttgart

Alle Rechte vorbehalten. Kein Teil dieser Zeltschrift darf ohne schriflliche Genehmigung des Veriages vervielfältigt oder verbreitet werden. Unter dieses Vorbehalt fallt insbesondere die gewerbliche Vervielfälltigung per Kopie, die Aufnahme in elektronischen Datenbanken und die Vervielfalligung auf CD-ROM und allen anderen elektronischen Datentrăgem.

Druck und buchbinderische Verarbeitung: Rosch-Buch, Scheßlitz Geduckt auf săurefreiem und chiorfrel gebleichtem Papier. ISTinted in Germany

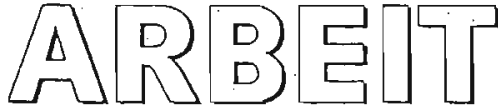

Zeitschrift für Arbeitsforschung Arbeitsgestaltung und Arbeitspolitik

\section{Jahrgang (2002) Heft 4}

\section{Inhaltsverzeichnis}

\section{Abhandlungen}

Ilse R. Schur

Expansion des Arbeitsmarkts im Dienstleistungssektor.

Haushaltsdienste für jedermannj-frau

Berndt Keller

Regulierung europäischer Arbeitsbeziehungen.

Das Beispiel Europäischie Aktiengesellschaft

\section{Alexander Stoll, Peter G. Richter}

Beeinflusst der Zentralisationsgrad einer Organisation

teambezogene Einstellungen und die Qualität der

Teamarbeit? Die Formulierung einer Hypothese

am Beispiel von Pflegeteams

Markus C. Pohlmann

Der Beitrag der Organisationsberatung zur Entwicklung der Organisation

Abstracts (English)

\section{Kurzbeiträge}

Andreas Burzik

Flow-Erfahrungen bei Orchestermusikern

Eine qualitative Studie über fördernde und

hemmende Bedingungen von Glückserfahrungen

im Arbeitsalltag professioneller Orchestermusiker

Michael Jonas, Marion Berner, Tabea Bromberg

Clusterbildung oder zufällige Zusammenballung?

Aspekte regionaler Entwicklungsprozesse am Beispiel

der Mikrosystemtechnik im Raum Dortmund 


\title{
Der Beitrag der Organisationsberatung zur Entwicklung der Organisation
}

\begin{abstract}
Trotz der einfach erkennbaren Vorteile von Organisationsberatung gegenüber anderen Beratungsformen ist bis heute unklar geblieben, wie genau und unter welchen Bedingungen Organisatiónsberatung funktioniert. Der Beitrag gibt Antworten darauf, welche Interaktionsform einen Organisationsberatungsprozess koristituiert, und inwieweit sie organisationale Strukturänderungen herbeifüren kann. Dabei zeigt er am Beispiel der ostdeutschen Beratungslandschaft auf, dass der sanfte, nicht direktive Weg der Organisationsbèratung harten Gestaltungsrestriktionen ausgeselzı war. Durch Organisationsberatung konnten nicht ohne weiteres wirksame Änderungseffekte erzielt werden. Organisationsberatung erwies sich, anders als ihre Vertreter postulieren, in der osideutschen Strukturkrise als ebenso enttäuschungsreiche wie voraussetzungsvolle Form von Beratung. Es zeigte sich, dass Organisationsberatung nur begrenzt krisentauglich war. Dabei galt: Je professionalisierter die Organisation war, des to eher schuf sie wirksame Gestaltungsformen von Beratung und desto eher gelang es ihr, durch O'rganisationsberatung angeregte Strukturänderungen in der Organisation zu stabilisieren.
\end{abstract}

\section{Problemstellung: Organisationsberatung und die Abkehr von der klassischen Modernisierung}

In der Organisationssoziologie ist das Thema der Organisationsberatung in den letzten Jahren immer wichtiger geworden. Im Zuge einer stärkeren Betonung von Organisationsentwicklung stellte sich die Frage, in welcher Weise man der Entwicklung von Organisationen eine Richtung geben könne. Sie stellte sich umso dringlicher, als zunehmend erkannt wurde, dass einfache Interventions- und Steuerungstheorien der Komplexität des Phänomens „Organisatíon" nicht Herr werden konnten. Die Idee zieldeterminierter korporativer Akteure war mit ein Grund dafür, dass die Enttäuschungen bei allen, die sich an Jer Veränderung und Entwicklung von Organisationen versuchten, so gross waren. So berichtele „New Leaders"; dass US-amerikanische Unternehmen Anfang/Mitte der neunziger-Jahre mehr als $200 \mathrm{Mrd}$. US-Dollar für Veränderungen, Umschulungen oder andere Formen der Überprüfung ihrer Organisation ausgegeben haben. Weniger als 20\% der Manager, die diese Ausgaben zu verantworten hatten, waren jedoch mit den Ergebnissen zufrieden - ein Verhälınis, das nach einer von Arthur D. Little durchgeführten Befragung von Führungskräften Ende der neunziger-Jahre sogar auf 16\% gefallen ist (zit. in Laszlo 1999, 32).

Aber trotz dieser Enttäuschungen folgt die Praxis des Organisierens immer noch gerne, so scheint es, dem Leitbild der klassischen Modernisierung. Zu diesem gehören die Idee einer

* unter Mitarbeit von Holger Gerlach.

Arbeit, Heft 4, Jg 11 (2002), S. 329-343 
einfachen intentionsgemässen und, direktiven Vèränderung von Organisation, die Idee einer möglichen korporativen ,Vereinnahmung "von Handlungsrationalitäten und einer einfachen Übersetzung von individueller in organisationale Vernunft. Sie klammerl sich umso mehr daran, je deutlicher wird, wie wenig sich die Realität organisationaler Veränderungsprozesse diesen fügt. Für die Vertreter der Organisationsentwicklung, die häufig ohne Erfolg darauf hingewiesen haben, wie problematisch der Ideenhorizont der klassischen Modernisierung geworden ist, schien Organisationsberatung deswegen als ein geeigneter. Weg, der Organisationsentwicklung eine Richiung zu geben, ohne die Fehler und Hlusionen der klassischen Modernisierung zu reproduzieren. Es entwickelte sich eine modernișierte Version der Orgânisationsberatung, mit der sich weder die Illusion der zielgerichteten „Durchgriffskausalität" noch die Vorstelfung direktiver Intervention mehr verband. Organisationsberatung sétzte vielmehr auf eine Interaktionsform, die auf Reflexion und Orientierung ausgerichtet ist und auf direktive Eingriffe verzichtet.

Trotz ihrer einfach erkennbaren Vorteile, ist aber bis heute unklar geblieben, wie genau und unter welchen Bedingungen Organisationsberatung funktioniert. Es blieb offen, ob und unter welchen Bedingungen Organisationsberatung in ihrer "Sanftheit" weit reichende, in ihrei zeitlichen Befristung nachhaltige Effekte erzielen kann? An dieser Frage setzen deswegen die folgenden Ausführungen an. Sie versuchen eine Antwort darauf zu geben, welche Interaktionsform einen Organisationsberatungsprozess konstituiert und inwieweit sie organisationale Strukturänderungen herbeiführen kann. Dazu ist im ersten Schritt notwendig zll erklairen, was wir meinen, wenn wir von Beratung, insbesondere von Organisationsberatung sprechen (1.). Im zweiten Schritt sollen am Beispiel der ostdeutschen Beratungslandschaft (2.) die Gestaltungsparameter für Organisationsberatung herausgearbeitet werden (3.) und gezeigl werden, inwieweit das im Beratungsprozess erzeugte Wissen als entscheidungsrelevant erạchtet wird (4.) und zu nachhaltigen Strukturiänderungen führt (5:)

\section{Zur Sozio-Logik von Organisationsberatung}

Es hat sich gezeigt, dass es sich nicht lohnt, den „Gemischtwarenladen " von Beratungsfirmen auf einen Nenner bringen zu wollen. Dies hat in einem Grossteil der Beratungsliteratur nur fiir Verwirrung gesorgt. Wenn wir hier von Beratung sprechen, sprechen wir von einer Interaktionsform, deren "clifferentia specifica" im Vergleich zu anderen Interaktionsformen klar zutage treten. So meinen wir keine Verkaufsinteraktion, bei der jemand einfach ein Plodukt, sagen wir Beratungssliteratur oder ein Gutachten, veräussert. Während die Kaüf/ Verkauf-Interaktion insbesondere auf Märkter auf ein punktuelles Ereignis zusammengezogen werden kann, hat Beratung einen prozessualen Charakter. Die Beratungsinteraktion ist dabei nicht durch eine minimale soziale Situation (wie z.B. bei Tausch auf Märkten möglich) gekennzeichnet, sondern durch eine vergleichsweise starke wechselseitige soziale Bezug nalume geprägt. Erst wenn Normen der Wechselseitigkeit Geltung erlangen können, sind die Voraussetzungen für eine Beratungsinteraktion geschaffen. Darüber hinaus kann Beratung als „Kontraktgut" nur in höchṣt unvollständiger Weise „Warenform " annehmen. Sie ist nicht als tauschbares Produkt mit genau spezifizierten Merkmalen materialişiert und als "Tauschgut" während der Interaktion und nachträglich veränderbar.

Mit einer Beratung meinen wir auch keine hierarchische Interaktion, in der uns jemand zwingt, cine Beratung zu machen oder noch schlimmer, seinen Rat zu befolgen (was diesen zu einer Anweisung machte). Zur Interaktionsform „Beratung" gehört sowohl die Freiwilligkeit im Einlassen auf diese Interaktionsform als auch die Freiheit in der Formulierung und Annahme" des Rates. Die Beratung, darauf hat Arimond bereits früh hingewiesen, lässt absolute Freiheit, den Rat anzunehmen oder nicht (Arimond 1.966, 186). Beide Seiten geben ihr Einverständnis zur Beratungsinteraktion. Ein Einverständnis, das (auch wenn es Kosten verursacht) jederzeit wieder entziehbar ist. Wichtig für diese Form der Wechselwirkung is daher die relative Unabhängigkeit der Akteure. Sie ist die Voraussetzung für die freie Einwilligung. Sie schafft eine Interaktionssituation, in der das Bezugsproblem der Beratung im Vordergrund stehen kann.

Beratung ist eine Interaktionsform, die auf verständigungsorientiertem Handeln basi ert. Damit ist gemeint, dass die Akteure sich darauf einlassen, ihre Handlungspläne intern aufeinander abzustimmen und ihre jeweiligen Ziele nur unter der Bedingung eines bestehenden:oder auszuhandelnden Einverständnisses über Situation und erwartete Konsequenzen verfolgen (vgï. dazu Habermas 1983/92, 144). Im Mittelpunkt von Beratung. steht ein „kommunikatives Handeln“, ohne dass deswegen eine teleologische, also zielgerichtete Handlungsstruktur verneint-wird (vgl. ebd.).'

Aufgrund der Verständigungsorientierung spielen Einfluss und Vertrauen innerhalb der Beratungsinteraktion eine grössere und Macht eine gerïgere Rolle. In Beratungen wird weder mit der Zuordnung von Vermeidungsalternativen (Luhmann) noch mit der Durchsetzung von Anweisungen gegen Widerstand (Weber) operiert. Viel eher zerstört Macht den Effekt-von Beratung. Man stelle sich den Unternehmer vor, Jer seinen Beratern die Ratschläge diktiert, die sie ihm zu geben haben. Oder umgekehrt den Berater, der der Unternehmer zwingt, einen Ratschlag anzunehmen. Beide Male wäre der Sinn der Beratung ad absurdum geführt und das für die Beratung zentrale Element der ,Überlegung“ eliminiert. Eine Beratung findet also idealtypisch im herrschaftsfreien Raum statt, d.h. es findet über ein freies Vertragsverhältnis von Akteuren hinaus keine dauerhafte Zuweisung von Anweisungsbefugnis von „Untergebenen" auf "Übergeordnete" statt. Im Falle von Beratung zersiören Macht und Herrschaft das für diese Interaktionsform notwendige Vertrauen. Dass Berate und Beratene Interessen haben, die nicht deckungsgleich sind, ändert daran nichts. Macht ist deshalb nicht in den Mittelpunkt der Analyse des Interaktionssystems zu stellen, sondern in den Mittelpunkt der Analyse seines organisationalen Kontextes. Hier gewinnen „herrschaf tskritische" Analysen an Relevanz (Iding 2000, 11; Pongratz 2000, 54).

Jede Beratung zielt, dadurch ist ihre Leistung bestimmt, auf eine Enweiterung des Problem- und des Handlungshorizontes des Beratenen. Gemeint ist damit, dass sie nicht mit Vorgaben operiert, die keinen Spielraum mehr lassen, die Handlungshorizonte verengen. Beratung ist als Interaktionsform immer eine Verständigung über Alternativen, Auswahlen, Folgen und Nebenfolgen von Entscheidungen.

Dies ist nach Habermas auch keine Voraussetzung für Verständigung bzw, konununikatives Handeln. Aber Dies ist nach Habermas auch keine Volaussetzung fur Verstandigung bzw, konunumikaitves Handeln. Aber
Verständigungsprozesse zielen nach Habermas auf Einverständnis - und genau dieses ist konstituierend fiir die Beratungsinteraktion. Einverständnis ist nicht gleichzusetzen mut Konsens. Es meint vielmehr ei ne Orientierung der Interaktion an ungeschriebenien, geteilten Regeln, so als ob eine Vereinbarung bestünde ( $\mathrm{vgl}$. dazu Weber 1922/8.56, 456 ff.). Dissens ist dauerbaft möglich, solange er die empirische Geltung der Regeln nicht untergräbt. Ein gutes Beispiel für dissensorientiertes Einverständnishandelu sind sicherlich die Aushan dJungen zwischen Arbeitgeber- und Arbeitnehmervertretungen. 
Abb. 1: Die Interaktionsform Beratung im Vergleich zu anderen Interaktionsformen

\begin{tabular}{|c|c|c|}
\hline KauffTausch & Beratung & \\
\hline punknuell & prozessual & \\
\hline $\begin{array}{c}\text { diskretionär (minimale soziale } \\
\text { Situation) }\end{array}$ & $\begin{array}{c}\text { relational (starke Normen der } \\
\text { Wechselseitigkeit) }\end{array}$ & \multirow[b]{2}{*}{ Hieranchie. } \\
\hline finit & nachträglich verändertbar & \\
\hline \multirow[t]{3}{*}{ (Gutachten etc.) } & Freiwilligkeit & Anweisung \\
\hline & redalive Autononie & Abhängigkeit \\
\hline & Einfluss/Vertrauen & Macht \\
\hline Stellvertretung & $\begin{array}{l}\text { Erweiterung des } \\
\text { Handlumgshorizontes }\end{array}$ & Verengung des Horizontes \\
\hline für Anclere handeln & selbat handeln & \multirow[t]{2}{*}{$\begin{array}{l}\text { ("Expertenberatung", Inhouse } \\
\text { Consulting, Co-Management) }\end{array}$} \\
\hline substitudiv & subsidär & \\
\hline $\begin{array}{l}\text { Entscheidungen für Aundere } \\
\text { treften }\end{array}$ & $\begin{array}{l}\text { Verständiging iuber } \\
\text { Entscheidungen }\end{array}$ & Belehrung \\
\hline \multirow{5}{*}{$\begin{array}{l}\text { (Umselzung von } \\
\text { Problemlösungen) }\end{array}$} & normorientiert & normgebunden \\
\hline & Sourveränität & "Unmündigkeit" \\
\hline & anderes Wissen & superiores Wissen \\
\hline & Selbst-/Themäbeobachtung & Wissen/Lnwissen \\
\hline & Experte/Experte & Experte/Laie \\
\hline
\end{tabular}

Mit Beratung ist auch keine Stellvertretungs-Interaktion gemeint, in der der Ratgeber seinen Rat gleich selbst in die Tat umsetzt (Sonst wäre eine Eheberatung eine sehr unstrittene Sache!). Die Beratenen wollen oder müssen handeln. Deswegen braúchen sie Beratung. Beratung ist eine Interaktionsform, bei der das Heft des Handelns bei den Beratenen bleibt. Das Handeln bleibt selbstbestimmt. Das heisst, die Beratung ist subsidiär, als Hilfe zur Selbsthilfe ausgelegt (vgl. dazu auch: Brem-Gräser 1993, 15; Willke 1994, 30 ff.). Es werden keine Entscheidungen für andere getroffen.

In dieser Verneinung des Stellvertretungshandeln gleicht die Beratungsintèraktion am chesten der Weiterbildung oder der Erziehung. Doch unterscheidet sie sich auch von der "Belehrung" in signifikanter Weise. Pädagogen formulieren anders als Berater einen allgemein verpflichtenden Anspruch, ihren Inhalten zu folgen (Normgebundenheit). Noch wich- tiger aber ist, dass Beratung (anders als Erziehung) Mündigkeit, Selbständigkeit und ein „Expertentum“", zumindest aber eine „Souveränität in eigenen Belangen" auf der Seite der zu Beratenden voraussetzt. Wenn diese Voraussetzung verletzt wird, gerät die Beratung in eine Schieflage. Aus soziologischer Sicht dreht es sich nicht um die Unter- und Überlegenheit von Wissen, sondern um unterschiedliche Perspektiven. Der Effekt von Beratung liegt in einem Ausnutzen der Differenz von Selbst- und Fremdbeobachtung begründet (Luhmann 2000 etc.). Dabei ist der Klient keineswegs der „Hörige" oder "Schutzbefohlene", wie es die Ursprung sbedeutung des Wortes will, sondern selbst Experte seines Betriebes (oder gar der Betrieb selbst). Eine Beratungsbeziehung ist also de facto eine Experten-Experten-Beziehung. Die wechselseitige Anerkennung als Experten ist dabei ein zentrales Element der Beratung.

Organisationsberatung meint vor diesem Hintergrund, dass mithilfe der Interaktionsform „Beratung" nicht nur individuelle, sondern organisationale Veränderungsprozesse angeregt werden söllen. Beratung setzt damit am Entscheidungssystem der Organisation an und versucht hier, eine Änderung von Entscheidungsprämissen zu bewirken. Organisationsberatung versucht also, das anhand der Differenz von Selbst- und Fremdbeschreibung erzeugte Wissen in das Entscheidungssystem der Organisation einzuspeisen und diese dadurch zur Selbständerung von Entscheidungsprämissen anzuregen. Erst wenn dies gelingt und die Organisation in einem auf Regeländerung bezogenen Modus lernt (Agyris/Schön 2000), kann Organisationsberatung nachhaltige Effekte erzielen. Sie kann dabei vom Ressourcenpool der Organisation profitieren, muss sich aber zugleich auch den Problemen organisierter Kollektivität stellen. Organisationsberatung ist daher sowohl von den Chancen, die sie hat, als auch von den Problemen, die sie meistern muss, eine potenzierte Form von Beratung.

Dabei wird seit den siebziger Jahren die Organisationsberatung als ein „Königsweg“ der Organisationsgestaltung ausgewiesen, der durch Überzeugung, indirekte Einflussnahme und Anregung zur Selbständerung einem zielorientierten Wandel der Organisation auf die Sprünge helfen könne. Das ältere Konzept von Beratung, so kann man mit Luhmann formulieren, hatte die Beratungsaufgabe auf die Planung der Entscheidungsprämissen und entsprechende Interventionen bezogen. „Dies wird jedoch fraglich (oder jedenfalls prinżipiell erfolgsunsicher)", so Luhmann, ,in dem Maße, als klar wird, dass die Stabilität eines dynamischen Systems in einer turbulenten Umwelt eine lose (und keine feste) Kopplung von Prämissen und Entscheidungen fordert" (Luhmann 2000, 433). Denn dem Berater (wie dem Leiter) fehlt es dann an „Durchgriffskraft". Die für das ältere Beratungskonzept konstitutive Trivialisierung von Organisation und Intervention ließ bei komplexen Problemen ein Gelingen der Intervention unwahrscheinlich erscheinen (vgl. dazu auch: Willke 19.94, 2ff.).

Der Berater ist in der Perspektive der ,Expertenberatung" (mehr noch als der Manager) ein Betriebsspezialist, der als Co-Manager die Probleme löst und in direktiver Weise nachhaltige Effekte erzielen kann. „Gemeinsam mit den Führungskräften des Klienten“, so Walger noch in den neunzigern, ,"öst der Experte komplexe Probleme und führt nachhaltige Veränderungen im Unternehmen herbei“"(Walger 1995, 5). Die Organisationsberatung ziel t hingegen darauf ab, auf Basis der "Eigenlogik" der Organisation zur Selbständerung anzuregen. Sie verzichtet dabei auf direktive Eingriffe und setzt statt dessen auf Einfluss, Überzeugung und Vertrauen mit dem.Ziel, den Problem- und Handlungshorizont in der Organisation zu erweitern.

Inwieweit dies in der Praxis der Organisationsberatung tatsächlich gelingt und Organisationsberatung zum zielorientierten Wandel der Organisation beitragen kann, soll uns im Folgenden genauer interessieren. Dabei orientiere ich mich an der Theorie organisationalen 
Wandels von Weick - einem der Begründer der modernen Organisationssoziologie in den USA. Sie hat den Vorteil, dass sie eine revidierte Evolutionstheorie, die auf Entwicklungsgesetze und Richtungsangaben verzichtet, auf Sinngebungsprozesse in Organisationen bezieht. Sie zielt auf die Erklärung von Strukturänderungen im Kontext der handelnden Aneignung von organisationalen Umwelten durch Akteure. Die Frage der organisationalen Strukturänderung erscheint dabei nicht von vorneherein - wie in der systemtheoretischen Variante - beantwortet, sondern von drei, in der Evolutionstheorie klassischen Schritten abhängig: Variation, Selektion und Stabilisierung bzw. Retention. Das bedeutet, nur wenn die Gestaltung der lose an die Organisation gekoppelten Umwelt des Beratungssystem zu einer Variation führt, die ausgewählt, also retrospektiv mit Sinn verbunden und als entscheidungsrelevant erachtet sowie als Produkt erfolgreicher Sinngebung, als neue Rationalitätsform, stabilisiert wird, können wir von einer nachhaltigen Strukturänderung der Organisation sprechen. Dabei dreht es sich um die Überlebens- und Änderungswahrscheinlichkeiten von organisationalen Sinnsystemen und nicht von Organisationen selbst. Diese zu behandeln wäre eine andere Frage, da eine Nicht-Änderung der Struktur für dás Überleben der Organisation genauso wichtig sein kann wie ihre Änderung. Mit Weicks Theorie organisationalen Wande]s ist u.E. ein handhabbares Schema gewonnen, mit dem die organisationalen Gestaltungs-, Selektions- und Stabilisierungsformen in Bezug auf Beratung analysiert und die Reich weite der möglichen organisationalen Beratungseffekte (ähnlich wie in der Theorie organisarionalen Lernens) rèlativ exakt bestimmt werden können.

\section{Der Testfall: Die Beratungspraxis in ostdeutschen Industrieunternehmen}

Als Testfall fiur die hier interessierende Frage, ob und unter welchen Bedingungen Beratung und insbesondere Organisationsberatung zur Wandel der Organisation beiträgt, habe ich die Beralungspraxis in ostdeutschen Industrieunternehmen - nach den Wirren der Treuhandphase und nach dem Abschluss der institutionellen Transformation - ausgewählt. Sie bot sich trotz einiger historischer, transformationsbedingter Besonderheiten - an, weil die Betriebe sich in der zweiten Hälfte der neunziger Jahre etabliert hatten und von da ạ vor allem mit nicht melxr transformationstypischen Krisensymptomen zu kämpfen hatten. Nach Ansich vieler Experten bot sich zu diesem Zeitpunkt eher ein ideales Betätigungsfeld für Berater Nicht nur der hohe Bedarf, sondern auch die hohen Qualifikationen des Pérsonals sowie die neu etablierten, unverkrusteten Organisationsstrukturen sprachen dafür. Zudem waren es häufig. Klein- und Mittelunternehmen mit komplexen Veränderungsproblematiken, die auch einen Großteil des sonstigen Beratungsgeschäftes in Deutschland ausmachen. Die Beraungspraxis in ostdeutschen Industrieunternehmen erscheint mir also zu diesem Zeitpunkt als keine zu schwere „Nagelprobe" für die Wirksamkeit, die Organisationsberalung unter Krisenbedingungen entfalten kann. In welcher Weise also wurde sie praktiziert und welche Chancen hatte sje, zu einem zielorientiertem organisationalen Wandel beizutragen?

Ich werde mich bei der Suche nach Antworten auf eine Reihe von empirischen Untersuchungen zur Beratungspraxis in Ostdeutschland im Zeitraum von Mitte bis Ende der neunziger Jahre beziehen, die vom Autor und Anderen ${ }^{2}$ am Institut für Soziologie der Friedrich-Schiller-Universität Jena durchgeführt wurden.

a) eine von 1996 bis 1999 durchgeführte Untersuchung von Unternehmensberatern und beratenen Unternehmen mit 12 Fallstudien;

b) eine 1997 durchgeführte schriftliche Befragung von 230 in Ostdeutschland tätigen Unternehmensberatern;

c) eine von 1995 bis 1997 durchgeführte Untersuchung von Management und Belegschaften in 10 ostdeutschen Betrieben ( $\varnothing$-Größe $289 \mathrm{Ma}$.) mit insgesamt 110 Interviews;

d) eine 1997 durchgeführce schriftlichen Befragung von insgesamt 220 Managern von ebenso vielen Industrieunternehmen in Ostdeutschland ( $\varnothing$-Größe $102 \mathrm{Ma}$.);

Zunächst zeigte sich anhand unserer Forschungsergebnisse, dass die These eines idealen Tätigkeitsfeldes für. Berater bestätigt wird. Fast die Hälfte der untersuchten ostdeutschen Unternehmen hatte Beratungen in Anspruch genommen, und davon haben wiederum fast die Hälfte (45\%) dies wiederholt getan. Die Beratungsfelder waren dabei vergleichsweise breit gestreut.

Tab. 1: Bereiche, in denen die Industrieunternehmen Beratung in Anspruch nahmen 1997/98

\begin{tabular}{|l|l|}
\hline \multicolumn{2}{|c|}{ Hauptsächliche Bereiche } \\
\hline Marketing, Vertrieb & $45,3 \%$ \\
\hline Finanzen & $44,4 \%$ \\
\hline Controlling & $38,1 \%$ \\
\hline Organisation & $37,0 \%$ \\
\hline Personal & $35,2 \%$ \\
\hline Produktion & $27,8 \%$ \\
\hline Techriologic. & $22,2 \%$ \\
\hline Logistik & $19,4 \%$ \\
\hline Qualitat & $16,4 \%$ \\
\hline Beschaffung & $14,8 \%$ \\
\hline
\end{tabular}

Mehrfaclnnennungen möglich ( $\mathrm{N}=103)$

Die Beratungsieistungen, die dabei in Anspruch genommen wurden, waren nach Angaben der Befragten zu mehr als einem Drittel der Fälle Leistungen die auf Co-Management, Management auf Zeit oder Beteiligung an der Umsetzung zielten (46,3\%). In etwas mehr als der Hälfte der Fälle beschränkte sich Beratung allerdings auf Analyse- und Konzeptentwicklungsleistungen, basierte auf subsidiären Beratungsvorstellungen und entsprach damit unserem Verständnis von Organisationsberatung: Organisationsberatung ließ sich auch in unseren Fallstudien in expliziter Abgrenzung zur umsetzungsorientierten „Expertenberatung "als eine dominante Orientierung der Beratungsunternehmen herausarbeiten. Konnte Beratung

2 Die Untersuchungen zur Unternehmensberatung wurden von Holger Gerlach, Andreas Hinz, Marek Krause Rudi Schmidt und dem Autor durchgeführt. 
also - und insbesondere Organisationsberatung - zum organisationalen Wandel in den ostdeutschen Unternehmen beitragen?

4 Die Gestaltung von Beratung: Variationsspielräume und ihre Grenzen

Um diese Frage zu beantworten, möchte ich im ersten Analyseschritt der organisationalen Beratungseffekte ëinige Anmerkungen zum Variationsspielraum machen, der der Organisationsberatung eröffnet wurde. Ich kann hier nicht die einzelnen neuen Variationen von Beratungswissen vorstellen, aber deutlich machen, welche Gestaltungsschemata oder kognitiven Landkarten der Akteure den Variationsspielraum in der Gestaltung der Organisationsberatung begrenzl und das Andocken des Beratungssystem an organisationale Entscheidungssysteme bereits in der Gestaltungsphase behindert haben. Dabei ist mit einem Gestaltungsschema (oder einer kognitive Landkarte) nur das verallgemeinerte und korrigierbare Muster gemeint, das die organisationalen:Erfahrungen gliedert und als erster Bezugsrahmen für Wahrnehmungen und Handlungen dient.

a) Dèr erste Bezugsrahmen der Handlungen war sehr klar durch eine Voran(g)stellung technologischer Fragen und eine Nachordnung organisatorischer definiert. Organisierung und Organisationsberatung. waren auf dieser Basis, auch wenn sie als wichtig erachtet wurden, immer nur periphere, nachgeordnete Elemente des ökonomischen Kalkills. Da die Prioritắtensetzung der Unternehmen im ,Wissenstransfer" auf der teuren Erneuerung der Technologie gelegen hatte, wurde die Organisationsgestaltung und insbesondere der Einbezug von externer Beratung dem daraufhin einsetzenden "Sachzwang" in der Mittelverwendung unterworfen (assimiliert). Organisationsberatung bewegte sich daher von den Gestaltungsparametern im Teufelskreis von zu wenig Zeit, zu hohem Problemdruck und zu hoher Problemkomplexität. Weit gehend ungeachtet der Komplexität des Problems - mehr als Dreiviertel der Unternehmen gaben an, dass sich die Problemstellung auf das gesamte Unternehmen bezog - dominierte eine Kurzform der Organisationsberatung Nur bei jedem vierten Betrieb umfasste die Beratungsdauer meh als vier Wochen. Und mehr als zwei Drittel der Berater gaben an, dass sie im Regelfall erst hinzugezogen wurden, als der Problemdruck bereits sehr hoch war. Dabei bestätigte sich, dass die hohe Punktualität und Temporalität der Beratung von vorneherein ihre Funktion als Organisationsberatung unterminierte.

b) Diese auf eine Kurzform der Beratung gerichteten Gestaltungsparameter hatte in der Hälfte der Fälle zur Konsequenz, dass auf einen über das Management hinausgehenden Einbezug weiterer betrieblicher Experten verzichtet wurde. Hinter der vordergründigen Kostenargumentation lag diesem Verzicht auf Beteiligung, dieser Konzentration auf Managementberatung ein Gestaltungsschema in der Organisation zugrunde, das von einer einfachen Übertragbarkeit von Managementwissen auf die Organisation durch Anweisung und Kommunikation ausging und deswegen eine weitergehende Beteiligung betrieblicher Experten als nachrangig erachtete. Die „, soziale Welt der Betriebe "blieb von der. Beratung deshalb oft unberührt. Nachhaltige Effekte der Beratung für die Organisation waren daher infrage gestellit.

c) Zu den Gestaltungsparametern der Beratung in ostdeutschen Industriebetrieben gehörte es auch, dass diese nicht immer freiwillig erfolgte, sondern auf Anweisung von Banken.
In solchen Fällen führten die Gestaltungsschemata der Organisation dazu, dass die Beratung im Regelfall nicht als solche anerkannt wurde. Sobald die Prämisse der relativen Autonomie verletzt wurde und die Macht der Banken ins Spiel kam, stieg die Misserfolgswahrscheinlichkeit der Beratung enorm. Sie wurde zu einer reinen Legitimationsveranstaltung und mündete in Schein-Beratungsinteraktionen, die keine wirklichen Effekte zeitigen konnten.

d) Darüber hinaus trat in der Krise die Prekarität einer Interaktionsform, die auf Hilfe zur Selbsthilfe setzt, klar hervor. In der Gestaltung des Beratungssystems kamen trotz einer Orientierung an Organisationsberatung immer wieder Gestaltungsschemata zum Vorschein, die auf Entlastung durch Stellvertretung zielten. Die prekäre Balance einer subsidiären Beratungsinteraktion war deswegen mit steigendem Problemdruck nicht immer zu halten.

e) Dabei spielte auch eine Rolle, dass der in der Krise besonders betonte, erfahrene betriebliche Pragmatismus ein Professionalitätsverständnis im Sinne der Organisations beratung, das dem Berater ein Mandat zur autonomen Problemdefinition zugesteht und sein Expertenwissen als anderes Wissen (als universelles, abstraktes, an den Standards der Zunft orientiertes Prozess- und Methodenwissen) akzeptiert, nicht immer zuließ. Beratungskonzepte und das Prozesswissen der Berater wurden nicht selten als „praxisferne Theorie" dechiffriert und als solche nicht anerkannt. Und während die eine Seite den Beratungsprozess durch die Besserwisserei und Belehrung der anderen blockiert sah, erachtete ihn die andere Seite durch die fachlichen Defizite des Managements erschwert.

Insgesamt lässt sich also am. Testfall der ostdeutschen Beratungslandschaft erkennen, dass in ihrer Gestaltung. unterschiedliche Schemata zur Anwendung gekommen sind, die den Variationsspiēlraum für. Organisationsberatung eng begrenzten und bereits die Etablierung einer ihr entsprechenden Interaktionsform nicht immer gelingen ließ. Welche Folgen hat die nun für die Selektion von in den Beratungssystemen erzeugten Wissen, für die retrospektive Sinngebung.und die Anschlussfähigkeit von Beratung für organisationale Entscheidungen

\section{Zur organisationalen Selektion: Retrospektive Sinngebung und} Anschlussfähigkeiten von Entscheidungen

Ein Großteil der mannigfaltigen Inputs in Organisationen, so Weick, bleibt "unberührt" Keineswegs immer interpretiert die Organisation also die im Gestaltungsprozess der Beratung produzierten Ergebnisse so, als ob eine relevante Entscheidung getroffen worden wäre. Erst wenn dies geschieht und Beratungswissen durch die retrospektive Sinngebung de Organisation als relevant selegiert wird, hat sich die Beratung als organisational anschlussfăhig erwiesen. Diese nach trägliche Sinngebung lässt sich in unserem zweiten Analyseschrit zunächst einfach erheben, indem man die Einschäızung des Ergebnisses der Beratung durch die Beratenen heranzieht. Sie ist deswegen bedeutsam, weil sich in den Fallstudien sehr klar gezeigt hatte, dass sich in den Organisationen, in denen die Beratung als Misserfolg eingeschätzt wurde, auch nichts an den Problem- und Handlungshorizonten sowie an den etablierten Organisationsstrukturen geändert hatte. Damit wissen wir zwar noch nichts über die organisationale Anschlussfähigkeit im Falle einer Erfolgseinschätzung, aber die Misser folgseinschätzung kann uns uns als Indikator dafür dienen, dass Beratungswissen in der Organisation folgenlos verpufft ist. 
Es zeigte sich, dass gemessen an diesem Indikator, die „Folgenlosigkeit" von Beratung kein seltenes Phänomien ist. Insgesamt rund $40 \%$ der Beraterien deklarierten die Beratung im Nachhinein als Misserfolg. Wenn sie, wie in Zweidrittel der Fälle angegeben, nicht den angänglichen Erwartungen entsprach, so war dies negativ gemeint: Die Beratenen waren enttäuscht. Und diese Enttäuschung schlug sich auch in der zu fast $80 \%$ geäußerten Absicht nieder, keine Beratungsleistungen mehr in Anspruch nehmen zu wollen.

\section{Tab. 2: Bewertungen von Beratungsleistungen durch die beratenen} Geschäftsführer 1997/98

\begin{tabular}{|l|c|}
\hline Die Beratung war eher ein Musserfolg. & $38,2 \%$ \\
\hline Die Beratungskosten waren der Leistung nicht angemessen. & $41,3 \%$ \\
\hline Die Beratung entsprach teilweise/gar nicht den Erwartungen. & $67,3 \%$ \\
\hline In näherer Zukunft keine Beratung mehr. & $77,5 \%$ \\
\hline
\end{tabular}

Mehrfachnennungen möglich (N=103)

Von den 120 Geschäftsführern, die keine Beratung in Anspruch genommen hatten, gaben zuderm fast die Hälfte an, dies aufgrund schlechter Erfahrungen mit Beratern nicht getan zu haben. In Bezug auf dieses hohe Maß an Unzufriedenheit mit Beratung, ist unser Testfall Ostdeutschland mitnichten ein Sonderfall. Beratung, das zeigen auch andere Untersuchungen, ist insgesamt ein enttäuschungsreiches Geschäft.

Tab. 3: Erfolgseinschätzungen von Unternehmensberatuingen durch die Klientel nach unterschiedlichen Untersuchungen 1993-2001

\begin{tabular}{|c|c|c|c|}
\hline Erfolgseinschätzungen von Beratungen & Proz. & $\mathrm{N}$ & Typ! \\
\hline $\begin{array}{l}\text { Beratung führte nicht zu einem unisetzungsfähigen Ergebnis. } \\
\text { (Lachnil/Muller 1993) }\end{array}$ & $24 \%$ & 161 & $\mathrm{KMU}$ \\
\hline $\begin{array}{l}\text { Beratung fulthrte nicht zu einer Umselzung der Ergebnisse. } \\
\text { (Kailer/Merker 1999) }\end{array}$ & $41 \%$ & 74 & KMU \\
\hline $\begin{array}{l}\text { Die Beritung war ... eher ein Misserfolg. } \\
\text { (Poh!mantu/Gerlach. 1999) }\end{array}$ & $38 \%$ & 104. & KMU \\
\hline $\begin{array}{l}\text { Die. Berntungsprojekte waren ... weniger erfolgreich. } \\
\text { (Fritz/Efrenberger 1998) }\end{array}$ & $31 \%$ & 141 & GU \\
\hline $\begin{array}{l}\text { Die Beritungsprojekte haben die Erwartungen ... } \\
\text { leilweise/gar nicht erfillt. (mannger magazin 200!) }\end{array}$ & $55 \%$ & 100 & GU. \\
\hline $\begin{array}{l}\text { Die Bcratungsprojekte wurden mil ... befriedigend oder } \\
\text { schlechter bewertet. (mantager magazin 200 } 1 \text { ) }\end{array}$ & $50 \%$ & 100 & GU \\
\hline $\begin{array}{l}\text { Mit der Beratung ... teils/leiis oder weniger zufrieden. } \\
\text { (Stöbe 1998) }\end{array}$ & $63 \%$ & 83 & Min. \\
\hline
\end{tabular}

I KMU =Klein- und Mittelunternehnen; GU=Großuncernehmen, Min. =Mlnisterien
Dabei gab es zentrale Faktoren, welche die Wahrnehmung von Beratung als Misserfolg deutlich erhöhten. Je kleiner der Betrieb, je höher das Honorar, je geringer die Beratungsdauer und Kontinuität, aber auch je weniger betriebliche Experten beteiligt waren, desto wahrscheinlicher war eine Misserfolgswahrnehmung der Beratenen.

\section{Tảb. 4: Misserfolgswahrnehmungen von Beratung $1997 / 98$}

\begin{tabular}{|l|l|c|}
\hline & Die Beratung war eher ein ... & Misserfolg \\
\hline Beratungsart & Expertenbefragung (Co-Management/Umsetzung) & $27,1 \%$ \\
& Organisationsberatung (Analyse, Konzepte etc.) & $46,5 \%$ \\
\hline Beratungsdauer & eine Woche & $61,5 \%$ \\
& bis einen Monat & $31,6 \%$ \\
& halbes Jahr und mehr & $18,8 \%$ \\
\hline Beteiligung & intensive Zusammenarbeit mit allen & $13,5 \%$ \\
& nur Zusammenarbeit mit Geschäftsführer & $45,5 \%$ \\
& keine Zusammenarbeit & $100 \%$ \\
\hline Initiator der Beratung & Bank & $54,5 \%$ \\
& eigene Initiative & $25,9 \%$ \\
\hline Beracungshonorar & gering & $20,0 \%$ \\
& hoch & $44,4 \%$ \\
\hline Betriebsgröße & klein (20-49) & $50,1 \%$ \\
& größer (über 200) & $7,1 \%$ \\
\hline
\end{tabular}

Am stärksten zu betonen ist aber, dass im Falle der Organisationsberatung eine Einschätzung der Beratung als Misserfolg wahrscheinlicher war als im Falle der so genanten Expertenberatung. Diese schuf, gemessen an diesem Indikator, ein geringes $\mathrm{Ma}$ an organisationaler Folgenlosigkeit, ohne dass damit jedoch bereits etwas über die Nachhaltigkeit ihres Beitrags zum zịelorientierten Wandel der Organisation gesagt wäre.

\section{Zur Stabilisierung von Änderungen: Die Perspektive organisationalen Wandels}

Diese Perspektive auf den organisationalen Wandel ist aber noch nicht hinreichend. Noch wissen wir nicht, unter welchen Bedingungen es zur organisationalen Anschlussfähigkeit kam und wann die.Selektion von Beratungswïssen' tatsächlich zur Ausbildung neuer Ratio nalitätsformen, und damit zu einem nachhaltigen Wandel der Organisation geführt hat: Es ist diese Frage der Stabilisierung von Änderungen, die jetzt im dritten Analyseschritt anhand der qualitativen Fallstudienergebnisse abschließend erörtert werden soll:

Anhand der Analyse von unterschiedlichen Organisationstypen und den damit korrespondierenden Gestaltungs-, Selektions und Stabilisierungsformen von Beratung ließen sich 
einige Regelnaufstellen, unter welchen Bedingungen Organisationsberatung in der Krise zur Veränderung von Organisationen beitragen konnten und unter welchen nicht

Immer dann, wenn in der Krise latente Regeln des „Durchwurstelns“ die Stabilisierung Immer da auf Änderungen bezogenen Erwar. bestimmen (,organisierte Anarchie'), nehmen die auf Anderungen bezogenen Erwartungsspielräume und Außenorientierungen bei den Akteuren in der Organisation sehr stark ab. Es wird kurzfristig reagiert, auf Basis einfacher Konzepte. Es kommt in der stab a kollektiv stabilisierten Selbstbezogenheit, die Organisation zu einer sehr emotionalen, kollektiv stabilisierten Selbstbezogenheit, die eine sehr restriktive Gestaltung von Organisationsberalung nicht nur nach sich zieht, sondern sich durch deren sich dann einstellenden Erfolgslosigkeit bestätigt sieht. Dass Organisationsberatung nicht weiterhilft, wird hier zur selbsterfüllenden Prophezeiung. Organisationsberatung nicht weiterhilf, wird hier zur selbstertlende Propheziung Entscheidungen orientieren kann Beratung in diesen Fällen nur, wenn sie keine ist, also in der Form des Co-Management praktiziert wird. Nur dann wird sie als Erfolg eingeschätzt.

Immer dann; wenn es zu einer einfachen Modernisierung der Organisation nach dem Irist (,klassisch-moderne Organisation"), sorgt die Krise für Rationalmodell gekonm sie Außenorientierungen einen starken Konser und Änderungsbereltschaflen der Organ̈ Ä in vielen Fällen nicht in organisationale Änderungsfähigkeit. Vielen Beschäftigten ist das Sanktionsrisiko für unkonventionelles Handeln und Fehlern bei ungeregelter Verantwor lungsïbernahme zu hoch und vielen Managern Anreizsysteme zur Förderung der Veranttungsïbernahme zu hoch und vielen Krise nimmt im Zusammenspiel dieser Handlungswortungsübernahme zu teuer. In der Krise nimmt im Zusamma el dieser HandungsrationalitäLen der Rückgriff auf stark regelorientierte Handlungsnormen zu. Mechanistirationalitaten desteutung und restringieren die Gestal (O) tungsmöglichkeiten der Organisationsberatung. Zwar entsienen vor diesem auch an Organisationsberatung anknüpfende Entscheidungsonentienngen. Sie konnen jedoch im Auseinanderfallen von individueller Änderungsbereitschaft und organisatio jedoch Än Auseing naler Änderungsfähigkeit nur selten als Strukturanderungen stabilisiert werden basierten Organisation. Deren Stabilisierungsmechanismen sind in der Krise auf eine basierten Organisation. Der Experimentalismus in Sachen Strukturänderung ausgerich tet. Ein Zitat soll dies verdeutlichen:

Und wir haben auch immer ganz bewusst gesagt; die festgeschnittene Struktur im Momien "Unis wirklich irgendwo statisch ist, sondern ich betrachte eine

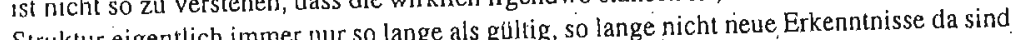
Struktur eigentlich immernur so an Und clas geht bei uns relativ schnell, dant

Gien Betrieben werden die großen Erwartungsspielräume des meist professionellen II diesen Betrieben werden die großen Personals für Strukturreformen genutzt. Die starke Außenorientierung der Akteure sorg zusammen mit der Kultur der Expertenge Rationalitätsformen des Beratungsprozesses. Er erweist sich bis hin zur Entfaltung neuer Rationalitatstormen as hoch anschlussfähig. Immer dann, wenn Organisationen mit experimentellen Strukuränderungen auf die Krise reagieren, entsteht zwischen einer experimentellen Außenturänderungen auf die Krise reagieren, orientierung mit hoher Veränderungsbereitschaft und dem Eing große Veränderungsmögberalung eine Wechselwirkung, die der
Tab. 5: Veränderungschancen durch Organisationsberatung nach Organisationstypen

\begin{tabular}{|l|c|c|c|}
\hline & Organisierte Anarchie & $\begin{array}{c}\text { Klassisch-moderne } \\
\text { Organisation }\end{array}$ & $\begin{array}{c}\text { wissensbasierte } \\
\text { Organisation }\end{array}$ \\
\hline $\begin{array}{l}\text { Gestaltung von Beratung } \\
\text { (Variation) }\end{array}$ & Organisationsberatung & Organisationsberanung & Organisationsberatung \\
\hline $\begin{array}{l}\text { Entscheidungsorientie- } \\
\text { rung (Selektion) }\end{array}$ & $\begin{array}{c}\text { Co-Management } \\
\text { "Expertenberatung" }\end{array}$ & Organisationsberatung & Organisationsberatung \\
\cline { 3 - 3 } $\begin{array}{l}\text { Strukruränderung } \\
\text { (Stabilisierung) }\end{array}$ & "Notoperation" & $\begin{array}{c}\text { "Expertenberatung" } \\
\text { Co-Management }\end{array}$ & Organisationsberatung \\
\hline Revolution (turn around) & "Notoperation" & "Notoperation" & nicht beobachtet \\
\hline
\end{tabular}

kursiv = Einfluss der Organisationsberatung auf den organisationalen Wandel

fett $=$ Einfluss/Notwendigkeit anderer Formen des Veriñderungsmanagements

\section{Resümee: Zum Zusammenhang von organisationaler Entwicklung und Organisationsberatung}

Unsere Ergebnisse zeigen sehr deutlich; dass auch Organisationsberatung von sich aus keine magische Qualität entfalten kann, die sie zu einem Allzweckmittel für organisationale Änderungen macht. Wir konnten im ersten Schritt der Analyse sehen, dass die sicherheitsgenerierenden Gestaltungsschemata der Organisation dafür sorgten, dass trotz einer Orientierung an Organisationsberatung diese in der Interaktionsform prekär und in ihrem Variations spielraum eng begrenzt bleibt. Dasselbe Bild zeigte sich in der Frage der Responsivität und die Rezeptionsfähigkeit der Organisationen für Beratungswissen. Trotz geleisteter Zahlungen war das Maß an organisational hergestellter ,Folgenlosigkeit" von Beratung, insbesondere von Organisationsberatung, beachtlich. Dahinter standen, das konnten wir im letzten Schric unserer Argumentation sehen, Stabilisierungsmechanismen der Organisation; die entwede in Form eines geheiligten Inkrementalismus jede Außenorientierung und Änderungsfähigkeit der Akteure zunichte machte oder in Form eines rationalen Konservatismus eher fü mechanistische Beratungsformen offen waren. Hoch anschlussfähig hingegen erwies sich Organisationsberatung vor allem in wissensbasierten Unternehmen, deren Stabilisierungsmechanismen auf einen experimentellen Relativismus eingestellı waren.

Organisationsberatung ist also eine hoch voraussetzungsvolle Form von Beratung, die nu r zur nachhaltigen Strukturänderung der Organisation beitragen kann, wenn privilegierte Gestaltungsbedingungen geschaffen werden. Je professionalisierter die Organisation, desto eher schafft sie Gestaltungsformen von Beratung, die ihre organisationale Anschlussfähigkeit mit organisieren können und desto eher gelingt es, Strukturänderungen zu stabilisieren . Aus unseren Ergebnišsen lässt sich ablesen, dass es eine Art Matthäus-Prínzip der Organisationsberatung gibt: Wer hat, dem wird gegeben und wer nicht hat, dem kann auch Organisationsberatung kaum helfen. Organisationsberatung ist nicht per se krisentauglich, sondern. nur, wenn sie ihre eigenen Voraussetzungen mit organisiert. Je unsicherer und krisenhafter der Beratungskontext eingeschätzt wird, desto höher sind, das haben wir gesehen, die Scheiternsrisiken von Organisationsberatung. Im Prokrustes-Bett der in der Krise zur 
Geltung kommenden Rationalitätskriterien (wie z:B.: Kurzfristigkeit, Kalkulierbarkeit und Pragmatismus) konnte sich Organisationsberatung als Katalysator organisationalen Wandels wicht allzu oft bewähren. Sie muss in solchen Fällen im Vorhinein Maßnahmen ergreifen, um ihre Veränderungswirkungen abzusichern. Sie muss die Vorberatungsphase bereits beratend gestalten und die Beteiligung betrieblicher Experten sowie die Selbstbeteiligung der Führungskräfte zur Voraussetzung der Durchführung machen. Vielleicht kann so die Überlegenheit der Organisationsberatung in der Frage der Nachhaltigkeit gegenüber der kurzfristig zufrieden stellenden Entlastung, die "Expertenberatung" verspricht, stärker zur Geltung kommen.

\section{Literatur}

Argyris, Chris, Donald A. Schőn (1999): Die lernende Organisation. Grundlagen, Methode, Praxis Stuttgart

Arimond, H. (1966): Zeitgemäße Berufsaufklärung. Pśychologische Beiträge 9. o.O.

Brem-Gräser, Luitgard (1993b): Handbuch der Beratung für helfende Berufe. Band 2. München/Basel 11. Aullage

Dahl, Edgar (1967): Die Unternehmensberatung. Eine Untersuchung ausgewähiter Aspekte beratende Tätigkeiten in der Bundesrepublik Deutschland. Meisenheim am Glan

Elfgen, Ralph, B. Klaile (1987): Unternehmensberatung: Angebot, Nachfrage, Zusammenarbei Stuttgart

Fritz, Wollgang, Jens Effenberger(1998): Strategische Unternehmensberatung. Verlauf und Erfolg von Projekten der Strategieberatung; in: DBW, 58, 1, 103-119

Iding Hermann (2000): Hinter den Kulissen der Organisationsberatung. Qualitative Fallstudien von Beratungsprozessen im Krankenhaus. Opladen

Habermas, Jürgen (1983/92): Moralbewusstsein und kommunikatives Handeln; in: Jürgen Habermas: Morallbewusstsein und kommunikatives Handeln. Frankfurt/M., 5. Auflage

Hruschka, Erna (1969): Versuch einer theoretischen Grundlegung des Beratungsprozesses; in: Eberhard Bay u.a. ( $\mathrm{Hg}$.), 16

Kailer, Norbert, Richard Merker (1999): Kompetenz in der Beratung kleiner und mittlerer Unternehmen - Defizite und Barrieren limitieren den Beratungserfolg. Berichte aus der Angewandten Innovationsforschung. Bochum

Lachnit, Laurenz, Stefan Müller (1993): Nutzung von. Unternehmensberatung in mittelständischen Unternebmen; in: Der Betriẹb, 46, 28, 1381-1389

Laszlo, Ervin (1999): Total Responsibility Management - Unternehmen in umfassender Verantwortung fithren lernen; in: André Papmehl, Rainer Siewers (Hg.): Wissen im Wandel. Die lernende Organisation in 21. Jahrhundert. Wien/Frankfurt, 23-34

Luhmann, Niklas (1984): Soziale Systeme. Grundriss einer allgemeinen Theorie. Frankfurt/M

Luhmann, Niklas (2000): Organisation und Entscheidung. Opladen/Wiesbaden

Manager Magazin (200i): Gewinner ohne Glanz. 7, 49-61

Micklethwait, John, Adrian Wookdridge (1998): Die Gesundbeter. Was die Rezepte der Unternehmensberater wirklich nützen. Hamburg

Pongratz, Hans J. (2000): System- und Subjektperspektive in der Organisationsberatung; in: ARBEIT $9,1,54-65$

Sclein, Edgar (1969): Process Consultation: Its Role in Organization Development. Reading, MA Schein, Edgar (2000): Prozessberatung für die Organisation der Zukunft. Der Aufbau einer helfenden Beziehung. Köln
Stơbe, Sybille (1998): Verwaltungsmodernisierung und Beratung: Ergebnisse einer Befragung; in: Ulrich Pekruhl (Hg.): Unternehmensberatung. Profil und Perspektiven einer Branche. Graue Reihe des IAT 1998-03, Gelsenkirchen, 47-57

Walger, Gerd (1.995): Idealtypen der Unternehmensberatung; in: Gerd Walger (Hg.): Formen der Unternehmensberatung. Systemische Unternehmensberatung, Organisationsentwicklung, Expertenberatung und gutachterliche Beratungstätigkeit in Theorie und Praxis. Köln, '1-18

Weber, Max (1922/85): Wissenschaftslehre. Hgg. v. Johannes Winckelmann, Tübingen, 6. erneut durchgesehene Auflage

Weick, Karl E. (1985): Der Prozess des Organisierens. Frankfur/M

Willke, Helmut (1994): Systemtheorie II: Interventionstheorie. StuttgarUJena

Anschrift des Verfassers:

Prof: Dr. Markus Pohlmann

Institut für Soziologie

der Friedrich-Alexander-Universität

Erlangen-Nürnberg

Kochstraße 4

D-9J054 Erlangen
Schlagwörter: Arbeitsorganisation/Gruppenarbeit/

Organisationsentwicklung, Beratung, Geographischer Bezug (regional), Industrie, Soziologie, Strukturwandel 